\title{
Some results on Heron triangles
}

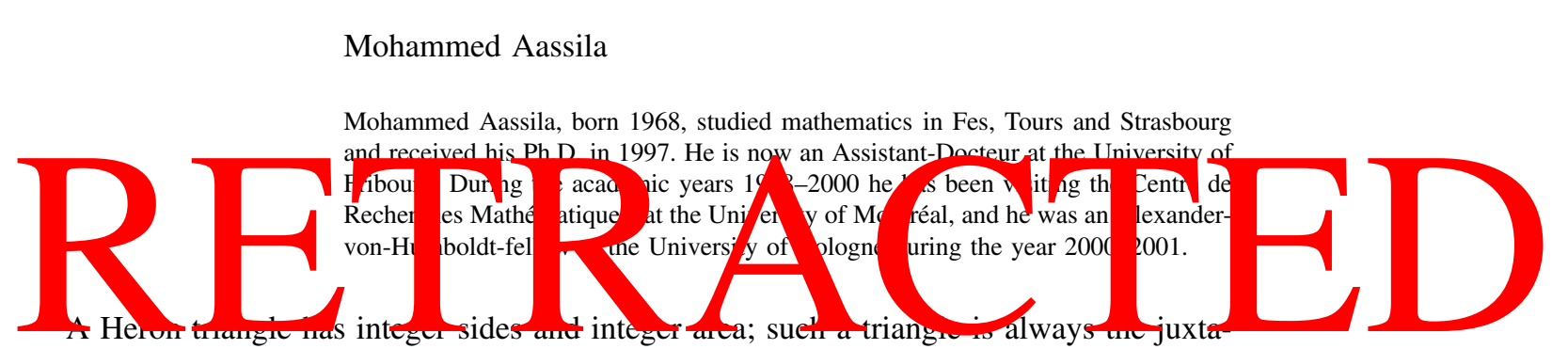

position (in a positive or negative sense) of two pythagorean triangles along a common leg. The common leg may or may not be an integer, as in the triangles $(13,14,15)$ and $(5,29,30)$ respectively. There are still several nice open problems concerning Heron triangles. For example, it is not known if there exist Heron triangles having all medians integers, see $[1,3,4,5]$ for related problems and results.

In this note, we present some results on the existence of pairs of incongruent Heron triangles with the same area and with the same area and perimeter. We furthermore prove the existence of Heron triangles with prescribed inradius and circumradius. Throughout this note we denote by $a, b, c$ the sides of a triangle and $S$ its area. The letters $s, r$ and $R$ stand for the semiperimeter, inradius and circumradius respectively.

As a first result we prove the existence of pairs of incongruent Heron triangles with the same area. More precisely

Theorem 1 There are many pairs of incongruent Heron triangles having the same area.

Bekanntlich wird ein Tripel natürlicher Zahlen $(a, b, c)$ ein pythagoräisches Tripel genannt, falls ein rechtwinkliges Dreieck mit den Seiten $a, b, c$ existiert. In leichter Verallgemeinerung dazu versteht der Autor des nachfolgenden Beitrags unter einem pythagoräischen Dreieck ein rechtwinkliges Dreieck mit rationalen Seiten. Ein heronsches Dreieck ist nun ein Dreieck mit ganzzahligen Seiten und ganzzahligem Flächeninhalt. Es lässt sich elementar zeigen, dass jedes heronsche Dreieck durch Zusammenstellen zweier pythagoräischer Dreiecke entlang einer Kathete entsteht. Auf den folgenden Seiten werden auf elementare Weise einige interessante Eigenschaften heronscher Dreiecke hergeleitet. Zum Beispiel wird bewiesen, dass es eine abzählbar unendliche Familie von Paaren inkongruenter heronscher Dreiecke mit gleichem Flächeninhalt gibt. 
Proof. Let $\left(F_{n}\right)_{n \geq 0}$ be the Fibonacci sequence defined by $F_{0}=0, F_{1}=1$ and $F_{n+2}=$ $F_{n+1}+F_{n}$ for all integers $n \geq 0$. Then there are many pairs of incongruent Heron triangles having the same area $S=F_{n} \cdot F_{n+1} \cdot F_{n+2} \cdot F_{n+3} \cdot F_{n+4} \cdot F_{n+5}$. Indeed, let $u$ and $v$ be two positive integers with $u \geq 2$ and $v \geq 1$. The triangle $T(u, v)$ of sides

$$
a=u^{2}+v^{2}, \quad b=(u v)^{2}+1, \quad c=(u v)^{2}+u^{2}-v^{2}-1
$$

has area

$$
S=\sqrt{s(s-a)(s-b)(s-c)}=u v\left(u^{2}-1\right)\left(v^{2}+1\right) .
$$

It remains to show that one can choose the pairs $(u, v)$ in two different ways such that the corresponding triangles $T(u, v)$ are incongruent but have the same area. We choose

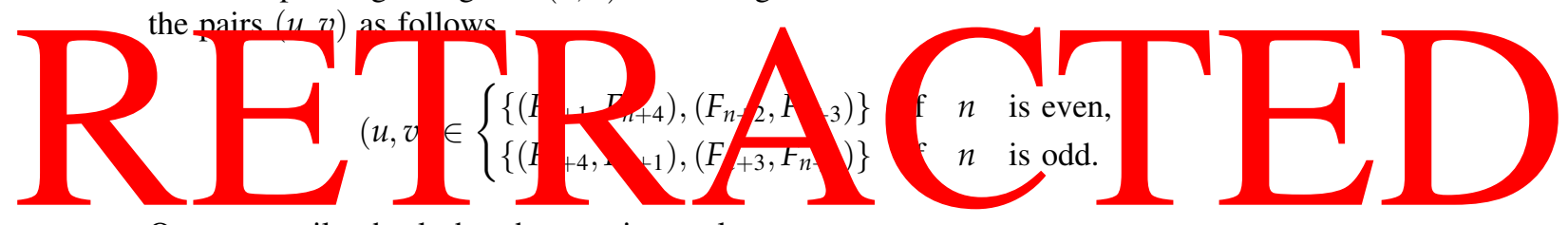

One can easily check that the area is equal to

$$
S=F_{n} F_{n+1} F_{n+2} F_{n+3} F_{n+4} F_{n+5} .
$$

In order to show that the triangle $T_{1}\left(F_{n+1}, F_{n+4}\right)$ is incongruent to $T_{2}\left(F_{n+2}, F_{n+3}\right)$, it suffices to notice that the shortest side of the triangle given by (1) is $a$. Hence, it is enough to prove that

$$
F_{n+1}^{2}+F_{n+4}^{2}>F_{n+2}^{2}+F_{n+3}^{2},
$$

or equivalently

$$
F_{n+2} F_{n+5}>F_{n} F_{n+3}
$$

and this is obviously true because $F_{n+2}>F_{n}$ for all $n \geq 0$.

As a second result in this note we have:

Theorem 2 There are infinitely many pairs of incongruent Heron triangles with the same area and perimeter.

Proof. Let $t \geq 1$ be any positive integer and let $T_{1}$ and $T_{2}$ be triangles of sides

$$
\left\{\begin{array}{l}
a_{1}=t^{8}+5 t^{6}+9 t^{4}+7 t^{2}+2 \\
b_{1}=t^{10}+5 t^{8}+10 t^{6}+10 t^{4}+6 t^{2}+3 \\
c_{1}=t^{10}+6 t^{8}+15 t^{6}+19 t^{4}+11 t^{2}+1
\end{array}\right.
$$

and

$$
\left\{\begin{array}{l}
a_{2}=t^{10}+6 t^{8}+14 t^{6}+16 t^{4}+9 t^{2}+2 \\
b_{2}=t^{6}+4 t^{4}+6 t^{2}+3 \\
c_{2}=t^{10}+6 t^{8}+15 t^{6}+18 t^{4}+9 t^{2}+1 .
\end{array}\right.
$$


Then, $T_{1}$ and $T_{2}$ are incongruent Heron triangles having the same semiperimeter

$$
s=t^{10}+6 t^{8}+15 t^{6}+19 t^{4}+12 t^{2}+3
$$

and the same area

$$
S=t\left(t^{2}+1\right)^{4}\left(t^{2}+2\right)\left(t^{4}+3 t^{2}+3\right) .
$$

We end this first part of the note by stating the following

Problem. Prove or disprove that for any positive integer $k \geq 2$ there exist $k$ mutually incongruent Heron triangles having the same area and semiperimeter.

As a third result, we have:

Theorem 3 Let $k \geq 1$ be a positive integer. Then, there exists a Heron triangle $T$ having $r=k$.

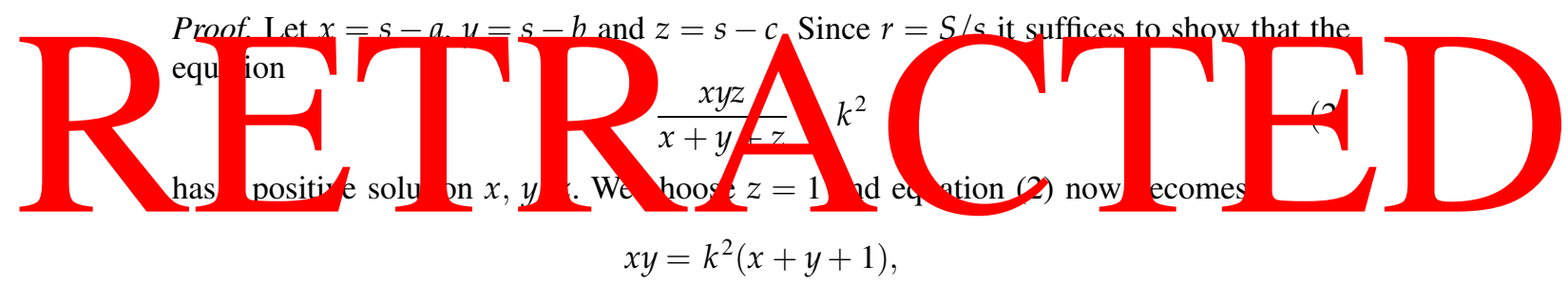

or equivalently

$$
x\left(y-k^{2}\right)=k^{2}(y+1)
$$

or also

$$
x=\frac{k^{2}(y+1)}{y-k^{2}} .
$$

Clearly, one may choose $y=k^{2}+1$ and then by (3) we have $x=k^{4}+2 k^{2}$. Hence, the triangle of sides

$$
a=k^{2}+2, \quad b=k^{4}+2 k^{2}+1, \quad c=k^{4}+3 k^{2}+1
$$

is a Heron triangle with $r=k$.

Finally, the last result of this note is:

Theorem 4 Let $p$ be a prime such that $p \equiv 1(\bmod 4)$. Then, there exists a Heron triangle $T$ having $R=p$.

Proof. Since $p$ is a sum of two squares (see Dickson [2]), we can write $p=u^{2}+v^{2}$. Then, the triangle of sides

$$
a=2\left(u^{2}+v^{2}\right), \quad b=2\left|u^{2}-v^{2}\right|, \quad c=4 u v
$$

is Heron and has $R=u^{2}+v^{2}=p$. Indeed, this follows immediately from the fact that the above triangle is right angled. 
Remark. It is clear from Theorem 4 that if $k$ is an arbitrary positive integer which is a multiple of $p$, then there exists a Heron triangle of circumradius $R=k$. To see this, it suffices to consider the triangle which is similar to the triangle given by (4) but whose sides are $k / p$ times longer.

Since almost every integer is divisible by a prime $p \equiv 1(\bmod 4)$, the result of Theorem 4 is "almost" true in general, however we claim that:

Conjecture. Let $p$ be a prime such that $p \equiv 11(\bmod 12)$. Then, there is no Heron triangle having $R=p$.

\section{References}

[1] J.R. Carlson: Determination of Heronian triangles, Fibonacci Quarterly 8 (1970), 499-506.

[2] L.E. Dickson: Introduction to the Theory of Numbers, University of Chicago Press, Chicago 1929.

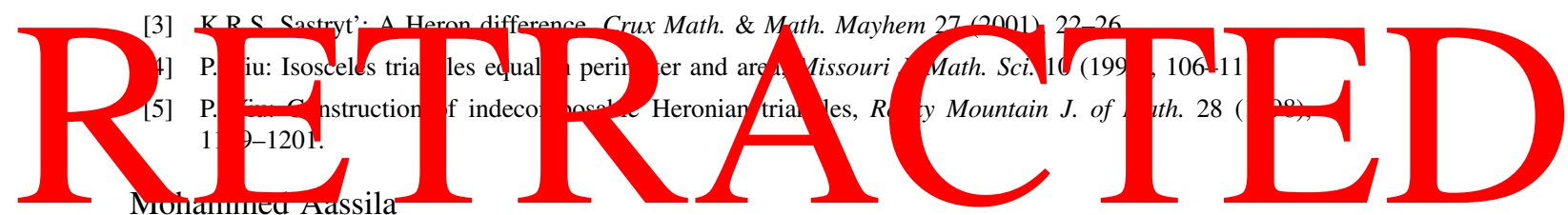

Institut de Mathématique

Université de Fribourg, Pérolles,

CH-1700 Fribourg, Suisse

e-mail: Mohammed.Aassila@unifr.ch

(D) To access this journal online:

(4P) http://www.birkhauser.ch 